\title{
PROPAGACIÓN in vitro DE SELECCIONES DE GUAYABO (Psidium guajava L.)
}

\author{
In vitro PROPAGATION OF GUAVA SELECTIONS (Psidium guajava L.)
}

\author{
L. Antonio Domínguez-Perales ${ }^{1}$, José L. Domínguez-Álvarez², \\ Serafín Cruz-Izquierdo' ${ }^{1}$, Amalio Santacruz-Varela' ${ }^{1}$, Alejandro Barrientos-Priego², \\ J. Saúl Padilla-Ramírez ${ }^{3}$ y Ma. Alejandra Gutiérrez-Espinosa ${ }^{\text {* }}$
}

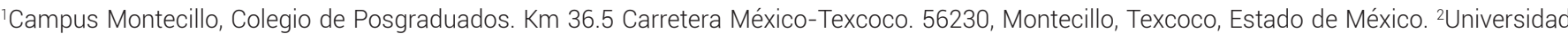
Autónoma Chapingo. Km 38.5 Carretera México-Texcoco. 56230, Chapingo, Estado de México. ${ }^{3}$ Campo Experimental Pabellón, Instituto Nacional de Investigaciones Forestales, Agrícolas y Pecuarias. Km. 32.5 Carretera Aguascalientes-Zacatecas. 20660, Pabellón de Arteaga, Aguascalientes.
\end{abstract}

*Autor de correspondencia (alexge@colpos.mx)

\section{RESUMEN}

La propagación in vitro mediante organogénesis directa de brotes de guayabo (Psidium guajava L.) constituye una alternativa para la rápida obtención de plantas productivas. El presente trabajo tuvo como objetivo optimizar un protocolo de regeneración in vitro a partir de ápices y segmentos nodales de cinco genotipos de guayabo con alto potencial comercial Paluma 12 (PM12), Paluma 6 (PM6), 17-06, Roja Exterior Redonda (RER) y Roja Chapeada Grande (CHRG). Se evaluó la adición de polivinilpirrolidona (PVPP) en el medio de cultivo como agente antioxidante. Los genotipos RER y CHRG presentaron menor número de brotes, con 10 y $0 \%$ de fenolización. En la fase de multiplicación de propágulos los mejores resultados fueron obtenidos cuando los brotes se desarrollaron en el medio Murashige y Skoog (MS) con el suplemento de $0.5 \mathrm{mg} \mathrm{L}^{-1}$ de BAP y con $30 \mathrm{mg} \mathrm{L}^{-1}$ de adenina para los genotipos PM6 y PM12, mientras que el genotipo 17-06 tuvo mejor desarrollo con la adición de $15 \mathrm{mg} \mathrm{L}^{-1}$ de sulfato de adenina. La adición de $40 \mathrm{mg} \mathrm{L}^{-1} \mathrm{de}$ sulfato de adenina representó el mejor tratamiento para los genotipos RER y CHRG. El enraizamiento se indujo satisfactoriamente mediante la incubación de los brotes durante $24 \mathrm{~h}$ en medio MS suplementado con $2.54 \mathrm{mg} \mathrm{L}^{-1}$ de AIA para el genotipo 17-06, con $1.34 \mathrm{mg} \mathrm{L}^{-1}$ de AIB para los genotipos PM6 y RER, y con 2.94 de AIB para los genotipos CHRG y PM12.

Palabras clave: Psidium guajava, organogénesis directa, optimización de protocolo, genotipos.

\section{SUMMARY}

In vitro propagation of guava (Psidium guajava L.) shoots by direct organogenesis is an alternative to quickly obtain productive plants. This work optimized a protocol for in vitro regeneration from apexes and nodal segments of five different guava genotypes with high commercial potential Paluma 12 (PM12), Paluma 6 (PM6), 17-06, Roja Exterior Redonda (RER) and Roja Chapeada Grande (CHRG). The effect of polyvinylpyrrolidone (PPVP) was evaluated as an antioxidant. Genotypes RER and CHRG showed low phenolization problems of 10 y $0 \%$, respectively. In the multiplication phase, genotypes PM6 and PM12 showed the best results when their shoots were grown on Murashige y Skoog medium (MS) supplemented with $0.5 \mathrm{mg} \mathrm{L}^{-1} \mathrm{BAP}$ and $30 \mathrm{mg} \mathrm{L}^{-1}$ adenine; meanwhile var. 17-06 developed better by addition of $15 \mathrm{mg} \mathrm{L}^{-1}$ of adenine sulfate. Addition of $40 \mathrm{mg} \mathrm{L}^{-1}$ of adenine sulfate resulted in the best treatment for vars. RER and CHRG. Rooting was induced by $24 \mathrm{~h}$ of shoot incubation on MS medium supplemented with $2.54 \mathrm{mg} \mathrm{L}^{-1}$ IAA for var. 17-06, while IBA at $1.34 \mathrm{mg} \mathrm{L}^{-1}$ induced rooting in cvs. PM6 and RER; IBA concentration had to be increased to $2.94 \mathrm{mg} \mathrm{L}^{-1}$ for better rooting in var. CHRG and PM12.
Index words: Psidium guajava, direct organogenesis, protocol optimization, genotypes.

\section{INTRODUCCIÓN}

México es uno de los principales productores y exportadores de guayaba (Psidium guajava L.) y sus derivados en el mundo. A pesar de esto, en los últimos años la superficie plantada y su producción han venido en decremento, ya que para 2013 la superficie plantada fue de 21,000 ha, mientras que para 2007 se reportaron 23,621 ha (SAGARPA, 2014). Esta situación se debe tanto a la alta heterogeneidad de las huertas como a la producción, además de que comercialmente se cultiva casi exclusivamente la variedad Media China (Padilla et al., 2002).

La generación y distribución de nuevos cultivares, en especial los que presenten pigmentación en sus frutos o sean de pulpa roja o rosada es una de las alternativas para cubrir las nuevas perspectivas en los mercados nacionales e internacionales, pues en los últimos años se ha incrementado la exigencia para la obtención de cultivares con altos contenidos de compuestos antioxidantes, como vitamina C, carotenoides y compuestos fenólicos (Carvalho et al., 2006; Chorilli et al., 2007).

La complementación de los métodos convencionales de propagación con herramientas biotecnológicas como el cultivo de tejidos constituye una alternativa para la reproducción. En el guayabo se han abordado diferentes métodos de propagación in vitro a través de la organogénesis, la embriogénesis somática y semilla sintética, desarrollados a partir de explantes de ápices, segmentos nodales y embriones zigóticos (Biswas et al., 2007; Chandra et al., 2004; Rai et al., 2007; Rai et al., 2010). No obstante, la propagación se ha caracterizado por una baja eficiencia en la obtención de plantas debido a la pérdida de explantes 
en la etapa inicial del establecimiento provocada principalmente por una alta oxidación fenólica y persistencia de contaminaciones bacterianas así como por un bajo coeficiente de multiplicación en etapas subsecuentes. Lo anterior ha conducido al desarrollo de protocolos para superar estas barreras biológicas (Concepción et al., 2005; Mishra et al., 2007a; Pérez et al., 2002). Recientemente se publicó acerca de la micropropagación del guayabo a partir de segmentos nodales en el cultivar Media China (Perales et al., 2016) sin embargo, no todos los genotipos de guayaba responden de la misma forma a los diferentes protocolos de micropropagación, por lo que se hace indispensable la generación de protocolos específicos para cada cultivar o genotipo de la especie.

Con base en lo anterior, la presente investigación tuvo como objetivo desarrollar un método de micropropagación en cinco diferentes genotipos de guayabo evaluados en Tabasco y Zacatecas, con alto potencial comercial para la región de los Cañones del estado mediante la evaluación del efecto de la polivinilpirrolidona PVPP como antioxidante y el tipo de explante en la fase de establecimiento in vitro, así como del efecto del uso de la adenina y del sulfato de adenina en la proliferación de brotes; además con el fin de promover la rizogénesis se evalúa la adición de auxinas en el medio de cultivo.

\section{MATERIALES Y MÉTODOS}

\section{Material vegetal}

Se consideraron cinco genotipos de guayabo: Paluma 12 (PM12), Paluma 6 (PM6), 17-06, Roja Exterior Redonda (RER) y Roja Chapeada Grande (CHRG). Estos genotipos fueron seleccionados de parcelas demostrativas en el municipio de Tabasco, Zacatecas. Las características que se utilizaron para su selección fueron tamaño y forma de fruto, color y grosor de pulpa, así como rendimiento en campo.

\section{Propagación in vitro de materiales de guayabo}

\section{Experimento 1. Establecimiento}

Se colectaron dos tipos de explantes de árboles de 2 a 3 años de edad creciendo en condiciones de invernadero, donde se mantuvieron las plantas en condiciones óptimas de fertilidad, humedad y sanidad mediante la aplicación constante de Curamicin ${ }^{\circledR}$ y Bactrimicin ${ }^{\circledR}$. La colecta se realizó durante los meses de septiembre a diciembre de 2012 y se utilizaron ápices de aproximadamente $2 \mathrm{~cm}$ de longitud y el primer nudo, los cuales se lavaron con detergente comercial durante 10 min y luego se enjuagaron en agua corriente. Después del lavado se realizó una desinfección superficial con hipoclorito de calcio $\left(\mathrm{CaCl}_{2} \mathrm{O}_{2}\right)$ a $3 \%$ que contenía tres gotas de Tween $® 80$ durante 10 min con agitación constante.

Los explantes ya desinfectados se colocaron individualmente en tubos de ensayo de $150 \mathrm{~mm} \times 25 \mathrm{~mm}$ que contenían medio sólido de establecimiento, constituido por medio basal MS (Murashige y Skoog, 1962) suplementado con sacarosa $30 \mathrm{~g} \mathrm{~L}^{-1}$, mio-inositol $100 \mathrm{mg} \mathrm{L}^{-1}$, tiamina 0.1

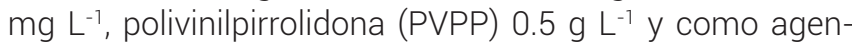
te solidificante se utilizó agar Merck® $6 \mathrm{~g} \mathrm{~L}^{-1}$. Después de establecer los explantes en el medio, se colocó la tapa de polipropileno y se sellaron los tubos e incubaron durante $10 \mathrm{~d}$ en condiciones de luz artificial indirecta (evitando el contacto directo con las lámparas), un fotoperiodo de 16 h luz, una intensidad de 2000 lux y una temperatura de 25 $\pm 2{ }^{\circ} \mathrm{C}$. La unidad experimental constó de 10 explantes, el diseño experimental fue completamente al azar con tres repeticiones, con dos factores a evaluar, genotipo y tipo de explante.

La evaluación se hizo de manera visual a los 10 d después de la siembra de los explantes. Se evaluó supervivencia (\%), pérdida por oxidación fenólica (\%) y contaminación por hongos y bacterias. La oxidación fenólica se cuantificó con base en dos aspectos: la oxidación o necrosamiento en el explante y la liberación de los fenoles del explante al medio de cultivo; para ello se usó una calificación de 1 a 3 [1 = baja, 2 = media (50\% del explante necrosado) y $3=$ alta (explante totalmente necrosado)] dependiendo de la cantidad de fenoles. El nivel tres se consideró como pérdida del explante.

\section{Experimento 2. Multiplicación de propágulos}

\section{Esta etapa de la propagación constó de dos fases:}

Fase 1. Efecto de la bencilaminopurina. La adición de la bencilaminopurina (BAP) al medio de cultivo se evaluó en su efecto en el desarrollo de nuevos brotes para los genotipos PM12, PM6 y CHRG (los genotipos RER y 17-06 se evaluaron en una investigación anterior). Se utilizaron brotes de tres nudos ya establecidos in vitro, los cuales fueron transferidos a frascos de cultivo que contenían $25 \mathrm{~mL}$ de medio semisólido de multiplicación, constituido por el medio basal MS suplementado con sacarosa $30 \mathrm{~g} \mathrm{~L}^{-1}$, mioinositol $100 \mathrm{mg} \mathrm{L}^{-1}$ y tiamina $0.1 \mathrm{mg} \mathrm{L}^{-1}$ medio al que se incorporó BAP en cuatro concentraciones diferentes (0.5, $1.0,1.5$ y $2.0 \mathrm{mg} \mathrm{L}^{-1}$ ). Los cultivos se incubaron durante 30 $\mathrm{d}$ en condiciones de luz artificial por lámparas fluorescentes, un fotoperiodo de $16 \mathrm{~h} \mathrm{luz}$, una intensidad de 2000 lux y una temperatura de $25 \pm 2{ }^{\circ} \mathrm{C}$.

La unidad experimental consistió en un frasco con seis brotes. El diseño experimental fue completamente al azar 
con cuatro repeticiones, y los factores fueron genotipo y la concentración de BAP. A los 30 d de iniciados los tratamientos se evaluó la longitud del brote inicial (LBI), el número de brotes por explante (NB), longitud de brotes (LB), el número de nudos del brote (NN), y el coeficiente de multiplicación (CM) que se calculó con la siguiente fórmula:

$$
\mathrm{CM}=\frac{\text { Cantidad de segmentos y brotes finales }}{\text { Cantidad de segmentos iniciales }}
$$

Fase 2. Efecto de adenina y sulfato de adenina. Debido a los bajos CM obtenidos en el experimento anterior y con base en información encontrada para otras especies (Bantawa et al., 2009; Nandagopal y Kumari, 2006), se optó por utilizar dos precursores de citocininas (adenina y el sulfato de adenina) para complementar el medio de cultivo. El medio de cultivo utilizado fue igual al del experimento anterior, adicionado con $0.5 \mathrm{mg} \mathrm{L}^{-1}$ de BAP (testigo).

El diseño fue completamente al azar, con arreglo de tratamiento factorial con el factor genotipo en cinco niveles, el tipo de precursor en dos niveles (adenina y sulfato de adenina) y el factor concentración de precursor en cinco niveles $\left(0,15,20,30\right.$ y $\left.40 \mathrm{mg} \mathrm{L}^{-1}\right)$. Los cultivos se incubaron durante $30 \mathrm{~d}$ en condiciones similares al experimento anterior. La unidad experimental consistió de un frasco con seis brotes, con cuatro repeticiones. La evaluación de las variables se realizó a los 30 d del inicio de los tratamientos y se consideraron las mismas variables que en el experimento anterior.

\section{Experimento 3. Enraizamiento}

Aquí se evaluó la respuesta del enraizamiento de brotes de los cinco genotipos de guayabo con diferentes reguladores de crecimiento de tipo auxínico. Se utilizaron brotes provenientes de la fase de multiplicación, los cuales fueron previamente homogeneizados en longitud $(1.5 \mathrm{~cm})$. Para inducir que los brotes formasen raíces adventicias, éstos se colocaron en frascos con $25 \mathrm{~mL}$ de medio semisólido de enraizamiento durante $24 \mathrm{~h}$, el cual estaba constituido por el medio basal MS complementado con sacarosa $30 \mathrm{~g}$ $\mathrm{L}^{-1}$, mio-inositol $100 \mathrm{mg} \mathrm{L}^{-1}$ y tiamina $0.1 \mathrm{mg} \mathrm{L}^{-1}$ al que se agregaron diferentes tipos y concentraciones de auxinas. Transcurrido el tiempo de inducción los brotes se transfirieron a frascos con medio basal MS mencionado.

El diseño experimental aplicado fue completamente al azar, con arreglo factorial de tratamientos en donde los factores fueron el genotipo en cinco niveles; el tipo de auxina en dos niveles (ácido 3-indolacético AIA y ácido 3-indolbutírico AIB) y la concentración de auxina en cuatro niveles $\left(0,6.6,14.5\right.$ y $\left.29 \mu \mathrm{mol} \mathrm{L}^{-1}\right)$. Los cultivos se incubaron durante 25 d en condiciones de luz artificial con lámparas fluorescentes, un fotoperiodo de $16 \mathrm{~h}$ luz, una intensidad de 2000 lux y una temperatura de $25 \pm 2{ }^{\circ} \mathrm{C}$. La unidad experimental consistió en un frasco con seis brotes, con tres repeticiones por tratamiento. Se evaluó la cinética de enraizamiento de los genotipos, con conteos diarios de la aparición de las raíces así como el conteo final del número y longitud de las raíces a los 25 d después de la inducción.

\section{Análisis estadístico}

Para el procesamiento de datos de los diferentes experimentos se aplicaron pruebas estadísticas para comprobar los supuestos de distribución normal y homogeneidad de varianzas. Al no cumplirse los supuestos, se aplicó la transformación de datos por medio de la formula $X^{\prime}=\sqrt{X+1}$ (donde $X^{\prime}=$ valor transformado; $X=$ valor original). Para las variables presentadas en porcentajes se hizo la trasformación de datos por medio de la formula $X^{\prime}=A R S E N \sqrt{X}$. Para desarrollar estos procedimientos se utilizó el paquete estadístico SAS versión 9.0. A los datos transformados y no transformados se les aplicó análisis de varianza (ANDEVA) y la prueba de comparación DHS de Tukey, con el paquete estadístico SAS versión 9.0.

\section{RESULTADOS Y DISCUSIÓN.}

\section{Experimento 1. Establecimiento}

La fenolización se caracteriza por un necrosamiento del tejido vegetal, y la emisión de coloraciones al medio de cultivo (Abdelwahd et al., 2008). En todos los tratamientos y explantes (ápices y nudos) aquí estudiados se mostraron estos síntomas, pero en diferentes intensidades. De acuerdo al ANDEVA, hubo diferencias significativas entre genotipos para las variables evaluadas (supervivencia \%, pérdida por oxidación \% y contaminación por hongos y bacterias), no así para los tipos de explante. También fue significativa la interacción genotipo por tipo de explante para las variables pérdida por oxidación y contaminación. Las aportaciones relativas de estos factores fueron de $60 \%$ para el factor genotipo, $19 \%$ para el tipo de explantes, mientras que la interacción de los factores tuvo una aportación de $21 \%$ sobre las variables evaluadas.

En la Figura 1 se muestran los porcentajes de fenolización de los explantes entre los genotipos de guayabo, y que la presencia de $0.5 \mathrm{~g} \mathrm{~L}^{-1}$ de PVPP indujo porcentajes de fenolización del nivel bajo. El genotipo CHRG presentó la mayor frecuencia de explantes con nivel bajo de oscurecimiento (93\%), seguido del genotipo RER que tuvo $87 \%$; estos dos genotipos fueron significativamente mejores que el resto de genotipos.

El porcentaje más alto de fenolización se dio en el genotipo PM6 donde 34 \% de los explantes mostró alto nivel 
de fenolización, en contraste con los genotipos CHRG y 17-06 que tuvieron 0 y $2 \%$ de explantes con alto nivel de fenolización, respectivamente.

Estos resultados concuerdan parcialmente con los obtenidos por Concepción et al. (2005) quienes reportaron que la adición de $0.5 \%$ de PVPP como antioxidante en el medio de cultivo fue efectivo para establecer explantes con niveles más bajos de fenolización en el establecimiento de yemas de guayabo Enana Roja Cubana. Sin embargo, este genotipo cubano solo alcanzó $24 \%$ de explantes con leve fenolización. Estos resultados ratifican el uso de PVPP como antioxidante que ha sido utilizado en guayaba (Ali et al., 2003; Concepción et al., 2005; Pérez et al., 2002); adicionalmente Concepción et al. (2005) mencionaron que el necrosamiento varía de acuerdo con el genotipo y que se puede revertir con el uso de PVPP.
La contaminación microbiana es uno de los factores limitantes más importantes para el inicio del cultivo in vitro vegetal (Krishna y Singh, 2007), que resulta en la muerte de los explantes o en retraso de su desarrollo. En la Figura 2 se presentan los porcentajes de supervivencia por tipo de explante y genotipo, donde los genotipos más afectados fueron el 17-06 con una supervivencia de $74 \%$ para los ápices y de $68 \%$ para los nudos, y PM6 con una supervivencia de $57 \%$ para los nudos. El genotipo RER tuvo el porcentaje de supervivencia más alto debido a que no mostró pérdidas de explantes por contaminación, además de ser uno de los que menos fenolización presentó, lo que lo hace un genotipo potencial para su manejo in vitro.

Al igual que para el caso de la oxidación, los explantes tuvieron un comportamiento uniforme. Las diferencias mostradas fueron atribuidas al genotipo y no al tipo de

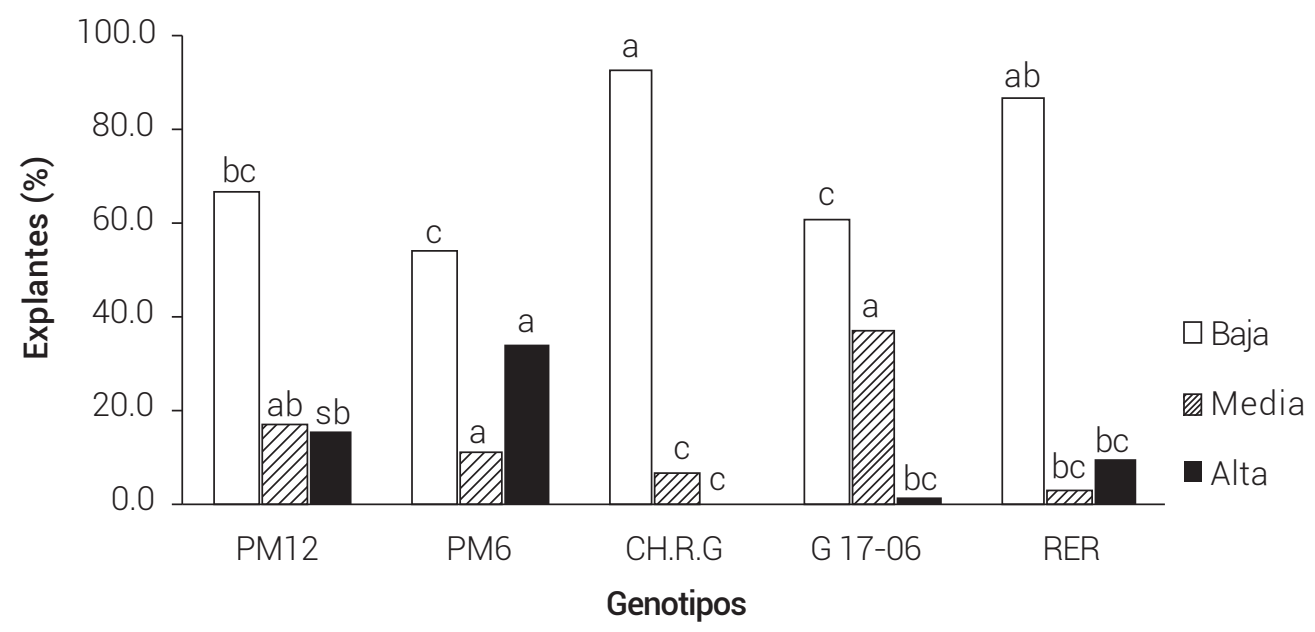

Figura 1. Fenolización de explantes en cinco genotipos de guayabo mediante el uso de PVPP como antioxidante, $10 \mathrm{~d}$ después de su establecimiento in vitro. Medias entre genotipos con letras iguales no son estadísticamente diferentes (Tukey, 0.05).

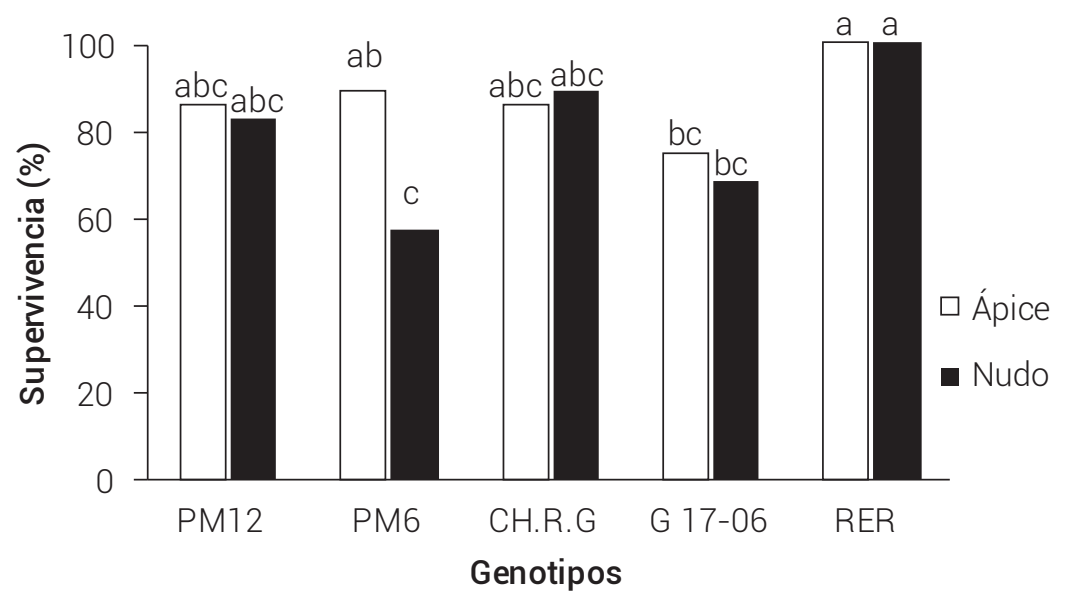

Figura 2. Supervivencia de dos tipos de explantes de cinco genotipos de guayabo a los $10 \mathrm{~d}$ después de su establecimiento in vitro. Medias con letras iguales no son estadísticamente diferentes (Tukey,0.05). 
explante, por lo que se pueden utilizar tanto ápices y nudos para el establecimiento de los cultivos in vitro. El uso del hipoclorito de calcio como desinfectante para estos genotipos sustituye al bicloruro de mercurio que es el desinfectante mayormente utilizado para guayabo (Concepción et al., 2005; Mishra et al., 2007a; Ocampo y Núñez, 2007), los procesos de oxidación son causados principalmente por el efecto abrasivo del agente desinfectante (Abdelwahd et al., 2008; Van Staden et al., 2006).

\section{Experimento 2. Multiplicación}

Fase 1. Efecto de bencilaminopurina. En el Cuadro 1 se presentan los valores promedio de las variables en estudio, para tres genotipos de guayabo. La longitud inicial del brote y número de nudos fueron estadísticamente igual para todos los tratamientos. Transcurridos $30 \mathrm{~d}$ de incubación la respuesta de los tres genotipos fue diferente para las concentraciones de BAP, y el genotipo CHRG respondió favorablemente a la concentración de $1.0 \mathrm{mg} \mathrm{L}^{-1}$, tratamiento en donde se observó la mayor longitud de brotes (de 1.04 a $1.37 \mathrm{~mm}$ ) con respecto al testigo. El genotipo PM12 presentó una mejor respuesta a la concentración de $0.5 \mathrm{mg} \mathrm{L}^{-1}$ ya que las variables número de brotes y coeficiente de multiplicación tuvieron los mayores valores (2.41 y 2.83, respectivamente). El genotipo PM6 mostró mayor respuesta a una concentración de $2.0 \mathrm{mg} \mathrm{L}^{-1}$ para la variable número de brotes (2.17); sin embargo, el coeficiente de multiplicación tuvo mejor comportamiento con una concentración de 0.5 $\mathrm{mg} \mathrm{L}^{-1}$ (2.05), lo que se debió a que los brotes tuvieron un mayor tamaño a una concentración de $0.5 \mathrm{mg} \mathrm{L}^{-1}$ y pudieron ser separados del brote inicial.

Los resultados indican que cada genotipo respondió de diferente manera a los reguladores de crecimiento, por lo cual es importante el establecimiento de protocolos específicos para cada genotipo, lo que confirma lo reportado por Papadatou et al. (1990) y Ali et al. (2003) en el sentido de que los niveles de citocininas son los más críticos para la multiplicación de muchos de los árboles tropicales, entre las cuales BAP es comúnmente la más usada en la propagación de guayabo.

Una exitosa regeneración in vitro depende del control de la morfogénesis, la cual se influye por diversos factores como el tipo de explante, la composición del medio de cultivo, los reguladores de crecimiento y el ambiente durante la incubación (Rai et al., 2010). El éxito de la micropropagación en especies arbóreas difíciles y recalcitrantes depende principalmente de la calidad de los explantes, calidad que está determinada en gran medida por el genotipo, el estado fisiológico del tejido y la época del año en que se colectaron (Giri et al., 2004).

Fase 2. Efecto de la adenina y el sulfato de adenina. En el Cuadro 2 se observan los valores promedio de las variables evaluadas para cinco genotipos de guayabo. Las diferencias estadísticas encontradas dependen en gran medida de la respuesta de los genotipos a los diferentes tratamientos. Para el genotipo PM6 su comportamiento promedio fue mejor

Cuadro 1. Efecto promedio de la concentración de bencilaminopurina en la multiplicación in vitro de brotes de guayabo de los cvs. Chapeada Roja Grande, PM12 y PM6, a los 30 d después de aplicados los tratamientos.

\begin{tabular}{|c|c|c|c|c|c|}
\hline Tratamiento (genotipo-mg L-1 $)$ & $\mathrm{LBI}(\mathrm{mm})$ & NN & NB & $\mathrm{LB}(\mathrm{mm})$ & $\mathrm{CM}$ \\
\hline CHRG $-0.5(T)$ & $14.3 \mathrm{a}$ & $2.46 \mathrm{a}$ & $1.04 \mathrm{c}$ & $4.4 \mathrm{c}$ & $1.29 \mathrm{ab}$ \\
\hline CHRG - 1.0 & $16.7 \mathrm{a}$ & $2.83 a$ & $1.37 \mathrm{bc}$ & $4.9 \mathrm{abc}$ & $1.29 a b$ \\
\hline CHRG - 1.5 & $15.7 \mathrm{a}$ & $2.95 \mathrm{a}$ & $1.37 \mathrm{bc}$ & $5.2 \mathrm{abc}$ & $1.21 \mathrm{~b}$ \\
\hline CHRG -2.0 & $15.2 \mathrm{a}$ & $2.83 \mathrm{a}$ & $1.08 \mathrm{c}$ & $3.4 \mathrm{c}$ & $1.12 b$ \\
\hline $\mathrm{PM} 12+0.5(\mathrm{~T})$ & $14.1 \mathrm{a}$ & $3.00 \mathrm{a}$ & $2.41 \mathrm{a}$ & $9.7 a b$ & $2.83 \mathrm{a}$ \\
\hline PM12 + 1.0 & $16.0 \mathrm{a}$ & $2.87 \mathrm{a}$ & $2.16 \mathrm{ab}$ & $8.6 \mathrm{abc}$ & $2.21 \mathrm{ab}$ \\
\hline PM12 + 1.5 & $15.0 \mathrm{a}$ & $2.66 \mathrm{a}$ & $1.87 \mathrm{abc}$ & $10.4 \mathrm{a}$ & $2.33 a b$ \\
\hline PM12 + 2.0 & $16.0 \mathrm{a}$ & $3.00 \mathrm{a}$ & $2.08 \mathrm{ab}$ & $8.1 \mathrm{abc}$ & $2.08 \mathrm{ab}$ \\
\hline $\mathrm{PM} 6+0.5(\mathrm{~T})$ & $14.0 \mathrm{a}$ & $2.61 \mathrm{a}$ & $1.61 \mathrm{abc}$ & $9.4 \mathrm{ab}$ & $2.05 a b$ \\
\hline PM6 + 1.0 & $14.3 \mathrm{a}$ & $2.77 \mathrm{a}$ & $1.94 a b c$ & $6.3 \mathrm{abc}$ & $1.89 a b$ \\
\hline PM6 + 1.5 & $13.2 \mathrm{a}$ & $2.44 \mathrm{a}$ & $1.94 \mathrm{abc}$ & $5.6 \mathrm{abc}$ & $1.22 \mathrm{~b}$ \\
\hline PM6 + 2.0 & $15.1 \mathrm{a}$ & $2.72 \mathrm{a}$ & $2.17 \mathrm{ab}$ & $5.2 \mathrm{abc}$ & $1.61 \mathrm{ab}$ \\
\hline C.V. & 12.00 & 8.18 & 21.34 & 34.76 & 35.64 \\
\hline
\end{tabular}

T : testigo; LBI: Iongitud del explante inicial; NN: número de nudos; NB: número de brotes; LB: longitud de brotes; CM: coeficiente de multiplicación; C.V.: coeficiente de variación. Medias con letras iguales en las columnas no son estadísticamente diferentes (Tukey, 0.05). 
cuando se desarrolló en medio de cultivo enriquecido con $30 \mathrm{mg} \mathrm{L}^{-1}$ de adenina, ya que el número de brotes aumentó de 1.61 a 3.54 con respecto al testigo, e igualmente el coeficiente de multiplicación aumentó de 2.02 a 3.5. El genotipo PM1 2 también se comportó mejor en presencia de la misma concentración de adenina, pues la longitud de los brotes aumentó de 97 a $180 \mathrm{~mm}$ con respecto al testigo, y el coeficiente de multiplicación aumentó de 2.83 a 4.31. El genotipo 17-06 presentó una mayor respuesta al sulfato de adenina como precursor de citocininas.

Con una concentración de $20 \mathrm{mg} \mathrm{L}^{-1}$ la variable número de brotes aumentó de 1.83 a 2.54 con respecto al testigo, mientras que la mayor longitud de brotes se obtuvo con una concentración de $15 \mathrm{mg} \mathrm{L}^{-1}$ de sulfato de adenina, y el coeficiente de multiplicación está altamente influenciado por las otras variables. El genotipo CHRG se vio afectado en el número de brotes cuando se añadió una concentración de $40 \mathrm{mg} \mathrm{L}^{-1}$ de adenina con valores por debajo del testigo, mientras que con una concentración de $15 \mathrm{mg} \mathrm{L}^{-1}$ de adenina se aumentó la longitud de los brotes de 44 a $92 \mathrm{~mm}$ y su coeficiente de multiplicación se elevó a 2.71 . Por otro lado, el genotipo RER mostró un mejor comportamiento promedio al desarrollarse en medio adicionado con sulfato de adenina $40 \mathrm{mg} \mathrm{L}^{-1}$, donde las variables NB, LB y el CM tuvieron los valores más altos $(2.15,105 \mathrm{~mm}$ y 3.40, respectivamente).

La adenina exhibe actividad similar a las citocininas sobre el desarrollo de brotes axilares, como lo describe Wróblewska (2012) en esquejes de Fuchsia hybrida; sin embargo, no muestra los efectos inhibitorios del enraizamiento típicos de las citocininas. Resultados similares se encontraron en la etapa de multiplicación in vitro de brotes de guayabo, pues al adicionar adenina o sulfato de adenina los brotes emitieron raíces y el desarrollo de los brotes no fue significativo.

La superioridad de BAP en la inducción de brotes se atribuye a la capacidad de los tejidos vegetales para responder a esta citocinina más rápidamente que a otros reguladores sintéticos, o de inducir la producción de hormonas naturales como la zeatina dentro del tejido (Malik et al., 2005). El control hormonal del crecimiento y desarrollo vegetal ha sido atribuido no sólo a la concentración sino a la sensibilidad propia de los tejidos en el sitio de acción (Gaspar et al., 2003). Otros aditivos del medio de cultivo que sirven para mejorar el crecimiento incluyen la sacarosa y el sulfato de adenina (Singh et al., 2002), que tienen efecto significativo en la multiplicación y elongación de brotes.

Aunque en la mayoría de los protocolos de propagación in vitro de guayabo los materiales vegetales muestran una mejor proliferación con el uso de BAP, Biswas et al. (2007) reportaron que el uso en combinación de citocininas como BAP y kinetina (Kin) aumentan la proliferación de brotes axilares a partir de segmentos nodales de guayabo, lo cual se debe al sinergismo de las citocininas; de igual manera, reportaron que el número de brotes por explante aumentó significativamente cuando el medio de multiplicación fue enriquecido con sulfato de adenina; así mismo mencionan que la adición de $0.1 \mathrm{mg} \mathrm{L}^{-1}$ de BAP, Kin y sulfato de adenina produjo en promedio 6.33 brotes de $3.23 \mathrm{~cm}$ de longitud, lo que representa seis veces más brotes que con el uso de las citocininas de manera individual.

Por otro lado, Singh et al. (2002) cultivaron brotes en medio adicionado con $0.2 \mathrm{mg} \mathrm{L}^{-1}$ de BAP y $10 \mathrm{mg} \mathrm{L}^{-1}$ de sulfato de adenina, para aumentar el número de brotes de guayabo cv. Allahabad Safeda. Sus resultados coinciden con lo observado en los cinco genotipos aquí evaluados, que con la adición de adenina y sulfato de adenina aumentaron significativamente tanto el número como la longitud de los brotes. El uso de sulfato de adenina por sí solo fue reportado por Bantawa et al. (2009) en Picrorhiza scrophulariiflora Pennel, en donde se mostró una tasa de multiplicación de 3 hasta 18 brotes por explante con 25 a 100 $\mathrm{mg} \mathrm{L}^{-1}$; sin embargo, la respuesta no cambió al usar bajas (25 $\left.\mathrm{mg} \mathrm{L}^{-1}\right)$ o altas concentraciones (400 $\mathrm{mg} \mathrm{L}^{-1}$ ), sino que fue el sinergismo con otras citocininas lo que condujo a una respuesta favorable. Altas concentraciones de BAP y en general de citocininas (>100 $\left.\mathrm{mg} \mathrm{L}^{-1}\right)$ provocan que en los nuevos órganos que se forman ocurran anormalidades como la generación de callo o el necrosamiento de los tejidos (Singh et al., 2002).

Nandagopal y Kumari (2006) en Chichorium intybus L. cv. Focus también reportaron que al adicionar sulfato de adenina en la etapa de multiplicación favoreció el aumento en la longitud de los brotes, al pasar de 2.66 y $3.50 \mathrm{~cm}$ a una longitud de 5.5 y $5.9 \mathrm{~cm}$ respectivamente en presencia de ese compuesto. De igual manera encontraron que para esta especie el mayor porcentaje de brotes se produjo con la adición de $1.5 \mathrm{mg} \mathrm{L}^{-1}$ de BAP + $0.5 \mathrm{mg} \mathrm{L}^{-1}$ de AIA y 0.25 $\mathrm{mg} \mathrm{L}^{-1}$ de sulfato de adenina. Por otro lado, para guayabo Biswas et al. (2007) y Singh et al. (2002) recomiendan la combinación de citocininas para mejorar la inducción de mayor brotación y aumentar su longitud.

\section{Experimento 3. Enraizamiento}

Se encontraron diferencias altamente significativas para los diferentes genotipos con una aportación relativa de 72 $\%$ para la variable longitud de raíces, mientras que para el tipo de auxina se encontró diferencia significativa en las variables número de raíces con una aportación relativa de $38 \%$ en porcentaje de enraizamiento. Por otro lado la concentración de auxina influyó significativamente solamente 
Cuadro 2. Efecto promedio de la concentración de adenina (A) y sulfato de adenina (S) en la multiplicación in vitro de brotes de guayabo de los cvs. PM6, PM12, 17-06, CHRG y RER, a los $30 \mathrm{~d}$ después de aplicados los tratamientos.

\begin{tabular}{|c|c|c|c|c|c|}
\hline Tratamiento (genotipo-precursor-mg L-1) & $\mathrm{LBI}(\mathrm{mm})$ & NN & NB & LB (mm) & $\mathrm{CM}$ \\
\hline PM6 Test. & $14.0 \mathrm{i}$ & $2.61 \mathrm{ij}$ & 1.61 efghijk & 9.4 & $2.05 \mathrm{fgh}$ \\
\hline A 15 & 28.2 & 3.77 & 3.02 & 11.0 & 3.31 \\
\hline A 20 & 26.8 & 3.45 & 2.88 & 9.3 & 3.22 \\
\hline A 30 & 25.3 & 3.48 & $3.54 \mathrm{a}$ & 9.3 & 3.51 \\
\hline A 40 & 28.0 & 3.28 & 2.57 & 7.8 & 2.88 \\
\hline S 15 & 30.2 & 3.68 & 3.14 & 6.9 & 2.20 \\
\hline S 20 & $33.5 \mathrm{a}$ & 4.26 & 1.76 & 6.1 & 2.46 \\
\hline S 30 & $32.4 \mathrm{a}$ & $4.34 \mathrm{a}$ & 3.05 & $5.8 \mathrm{fgh}$ & 2.65 \\
\hline S 40 & $30.4 a b c$ & 4.07 & 2.08 & 8.8 & 2.65 \\
\hline PM12 Test. & $14.1 \mathrm{i}$ & 3.00 ghij & 2.41 & 9.7 & 2.83 \\
\hline A 15 & 29.6 & 3.68 & 1.88 & 12.6 & 2.71 \\
\hline A 20 & 26.9 & $3.91 \mathrm{abcd}$ & 2.51 & 14.3 & 3.25 \\
\hline A 30 & $29.9 \mathrm{abcd}$ & 3.85 & 3.17 & $18.0 \mathrm{a}$ & $4.31 \mathrm{a}$ \\
\hline A 40 & 26.9 & 3.72 & 2.96 & 14.8 & 3.72 \\
\hline S 15 & 27.9 & $3.91 \mathrm{abcd}$ & 2.25 & 13.9 & 3.25 \\
\hline S 20 & 25.5 & 3.60 & $3.45 \mathrm{ab}$ & 14.3 & 4.22 \\
\hline S 30 & 26.5 & 3.88 & 2.79 & 14.1 & 3.74 \\
\hline S 40 & 26.9 & 3.77 & 3.25 & 15.8 & 4.14 \\
\hline 17-06 Test. & $16.0 \mathrm{ghi}$ & 2.83 hij & 1.83 & $4.1 \mathrm{gh}$ & $1.83 \mathrm{gh}$ \\
\hline A 15 & 22.7 & 3.54 & 2.40 & 9.9 & 2.88 \\
\hline A 20 & 22.6 & 3.74 & 1.99 & 8.4 & 2.42 \\
\hline A 30 & 23.2 & 3.54 & 2.00 & 8.1 & 2.31 \\
\hline A 40 & 26.7 & 3.51 & 2.00 & 10.6 & 2.80 \\
\hline S 15 & 25.5 & 3.54 & 2.17 & 11.8 & 2.68 \\
\hline S 20 & 26.6 & 3.71 & 2.54 & 5.9 & 2.08 \\
\hline S 30 & 25.6 & 3.75 & 2.10 & 8.3 & 2.28 \\
\hline S 40 & 27.2 & 3.77 & 2.28 & 6.2 & 2.02 \\
\hline CHRG Test. & $14.3 \mathrm{hi}$ & $2.46 j$ & 1.04 & 4.4 & $1.29 \mathrm{~h}$ \\
\hline A 15 & 25.9 & 3.00 & 1.40 & 9.2 & 2.71 \\
\hline A 20 & 28.0 & 3.12 & 1.12 & 9.2 & 2.56 \\
\hline A 30 & 25.2 & 3.04 & 1.40 & 8.0 & 2.56 \\
\hline A 40 & 29.6 & 3.16 & $0.64 \mathrm{k}$ & $4.1 \mathrm{gh}$ & 2.36 \\
\hline S 15 & 27.7 & 3.31 & 1.29 & 8.8 & 2.58 \\
\hline S 20 & 27.5 & 3.04 & 1.28 & 7.5 & 2.68 \\
\hline S 30 & 28.6 & 3.12 & 1.12 & 7.3 & 2.40 \\
\hline S 40 & $31.5 \mathrm{ab}$ & 3.32 & 1.28 & 9.1 & 2.76 \\
\hline RER Test. & 19.8 fghi & 3.46 & 1.26 hijk & $3.1 \mathrm{~h}$ & $2.26 \mathrm{efgh}$ \\
\hline A 15 & 22.1 & 3.30 & 1.40 & 5.5 & 2.35 \\
\hline A 20 & 20.2 & 3.05 & 1.95 & 8.5 & 2.85 \\
\hline A 30 & 23.0 & 3.10 & 1.35 & 9.3 & 3.10 \\
\hline
\end{tabular}


Cuadro 2. Continua.

\begin{tabular}{llllcr}
\hline Tratamiento (genotipo-precursor-mg L-1) & LBI $(\mathrm{mm})$ & NN & NB & LB $(\mathrm{mm})$ & CM \\
\hline A 40 & 23.0 & 3.20 & 1.45 & 8.5 & 3.00 \\
S 15 & 20.1 & 3.05 ghij & 1.45 & 10.3 & 2.85 \\
S 20 & 23.5 & 3.30 & 1.50 & 7.1 & 3.20 \\
S 30 & 23.2 & 3.25 & 1.85 & 8.2 & 3.20 \\
S 40 & 21.5 & 3.20 & 2.15 & 10.5 & 3.40 \\
C.V. & 13.21 & 9.05 & 26.27 & 31.86 & 19.29 \\
\hline
\end{tabular}

Test: testigo; LBI: longitud del explante inicial; NN: número de nudos; NB: número de brotes; LB: longitud de brotes; CM: coeficiente de multiplicación; C.V.: coeficiente de variación. Medias con letras iguales en las columnas no son estadísticamente diferentes (Tukey, 0.05).

sobre la variable porcentaje de enraizamiento con una aportación relativa de $43 \%$, mientras que la interacción de los factores genotipo y concentración de auxina tuvo una aportación de $14 \%$.

Para cualquier protocolo de micropropagación, un enraizamiento exitoso de brotes es un requisito para facilitar su aclimatación ex vitro (Pati et al., 2006). En guayabo la iniciación de las raíces ha sido estimulada por la incorporación de AIB sólo o en combinación con ácido 1-naftalenacético (ANA), aunque también se ha logrado la inducción en medio MS sólo (Rai et al., 2010). El tipo y la concentración de los reguladores de crecimiento empleados durante el establecimiento y multiplicación in vitro, así como las condiciones de desarrollo de los brotes y el genotipo son factores que influyen en los requerimientos de auxinas en el enraizamiento de guayabo (Ali et al., 2003).

Los diferentes genotipos respondieron de manera diferente al tipo y concentración de la auxina. En la Figura 3 se muestra que en ausencia de auxinas (testigo) se obtuvieron altos porcentajes de enraizamiento entre 72 y 92 $\%$, excepto para el cv. CHRG que fue de alrededor de $35 \%$. Estos resultados difieren de los obtenidos por Mishra et al. (2007b) quienes reportaron que para el cv. Allahabab Safeda no se logró inducir enraizamiento en ausencia de auxinas. El desarrollo de raíces en ausencia de reguladores de crecimiento puede ser debido a la síntesis de auxinas endógenas en cantidades necesarias para su desarrollo; estas concentraciones de auxina varían de acuerdo con el genotipo (Ali et al., 2003; Ocampo y Núñez, 2007).

El porcentaje de enraizamiento, además de ser influido por el genotipo, también es afectado por las condiciones del cultivo (Couprie et al., 2000). Para el guayabo cv. Pant Prabhart, Mishra et al. (2007a) obtuvieron $85 \%$ de brotes enraizados mediante el uso de una combinación de $0.4 \mathrm{mg}$ $\mathrm{L}^{-1}$ de AIB y $0.2 \mathrm{mg} \mathrm{L}^{-1}$ de ANA.

Generalmente el tiempo que se recomienda para hacer el trasplante a suelo de las plantas micropropagadas es de
28 a 30 d, sin considerar la especie o la longitud de las raíces; pero esto puede ocasionar daños durante el trasplante e incrementar el estrés, y dificulta la aclimatación (Thomas y Schiefelbein, 2001). Por ello es importante conocer el tiempo óptimo cuando se obtiene el mayor porcentaje de enraizamiento y los explantes han producido el número y longitud de raíces que faciliten su manejo sin afectar su supervivencia in vivo.

El tiempo requerido para enraizamiento difirió entre genotipos, y también se vio influido por el tipo y la concentración de la auxina. En el genotipo 17-06 las raíces empezaron a emerger a partir de los $3 \mathrm{~d}$ en presencia de AIB en una concentración de $5.9 \mathrm{mg} \mathrm{L}^{-1}$, sin alcanzar $100 \%$ de brotes enraizados; en cambio con el uso de AIA la respuesta fue a los 6 d y con la concentración de $3 \mathrm{mg} \mathrm{L}^{-1}$ se alcanzó $100 \%$ de brotes enraizados en $12 \mathrm{~d}$. Para el genotipo PM6 la respuesta de la inducción empezó entre los 6 y $7 \mathrm{~d}$ con el tratamiento de $1.3 \mathrm{mg} \mathrm{L}^{-1}$ de AIB, dónde se consiguió $100 \%$ de brotes enraizados a los $11 \mathrm{~d}$ (Figura 4).

En el genotipo CHRG los brotes empezaron a enraizar a los $5 \mathrm{~d}$ en una concentración de $2.5 \mathrm{mg} \mathrm{L}^{-1}$ de AlA y con el tratamiento de $3 \mathrm{mg} \mathrm{L}^{-1}$ de AIB se logró el $100 \%$ de brotes enraizados a los 14 d después de la inducción (Figura 5). El desarrollo de raíces en los brotes del genotipo PM12 se dio entre 7 y $8 \mathrm{~d}$ para los diferentes tratamientos; pero en ninguno de los tratamientos alcanzó $100 \%$ de brotes enraizados, ya que el valor más alto fue de $90 \%$ que se logró en presencia de $3 \mathrm{mg} \mathrm{L}^{-1}$ de AIB a los $12 \mathrm{~d}$. El genotipo RER fue el que más tardó en responder a la rizogénesis al haber iniciado entre los 9 y $10 \mathrm{~d}$. El tratamiento de $1.3 \mathrm{mg}$ $\mathrm{L}^{-1}$ de AIB fue con el que se obtuvo un mayor porcentaje de brotes formando raíces adventicias (90\%) a los $14 \mathrm{~d}$ de iniciados los tratamientos.

La emisión de raíces para los genotipos fue entre 6 y 8 $\mathrm{d}$ en promedio con los diferentes tratamientos, con excepción de RER, que fue entre 9 y 11 d. Estos resultados no concuerdan con los obtenidos por Mishra et al. (2007b) 


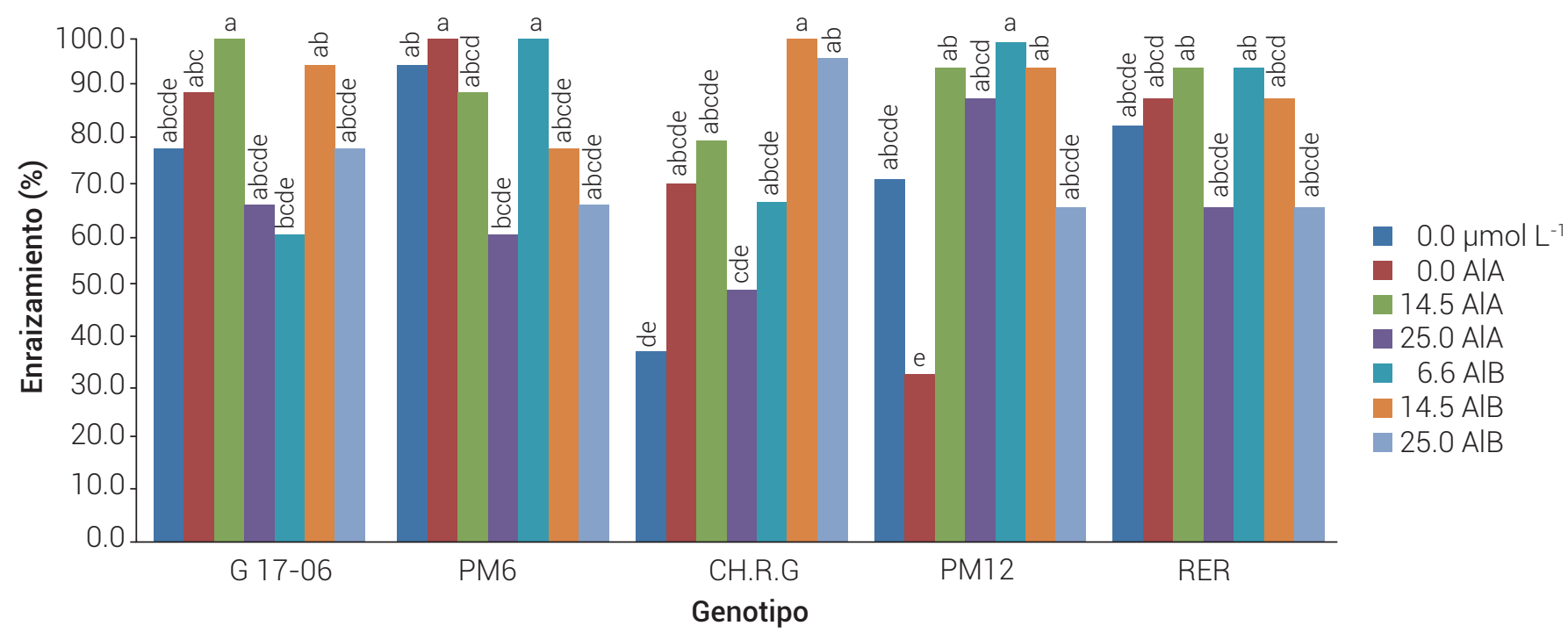

Figura 3. Efecto de la concentración $\left(0,6.6,14.5\right.$ y $\left.25.0 \mu \mathrm{mol} \mathrm{L}^{-1}\right)$ y tipo de auxina (AIA y AIB) sobre el enraizamiento in vitro de cinco genotipos de guayabo, a los $30 \mathrm{~d}$ después del inicio de los tratamientos. Barra con letras distintas, las medias que difieren significativamente según ANOVA y prueba de Tukey, con $\alpha=0.05$.

A)

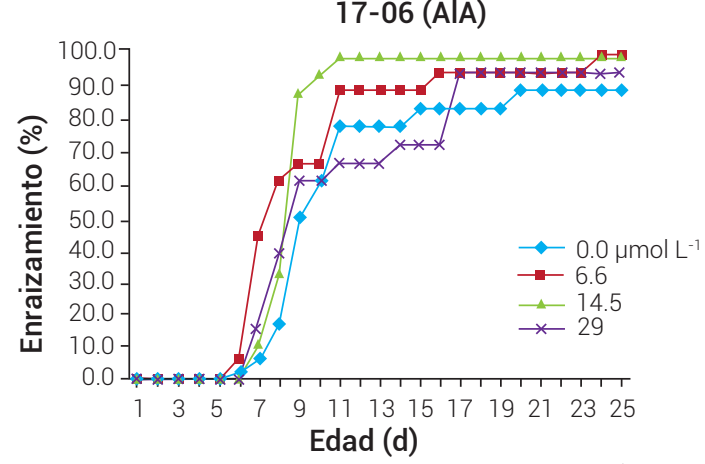

C)

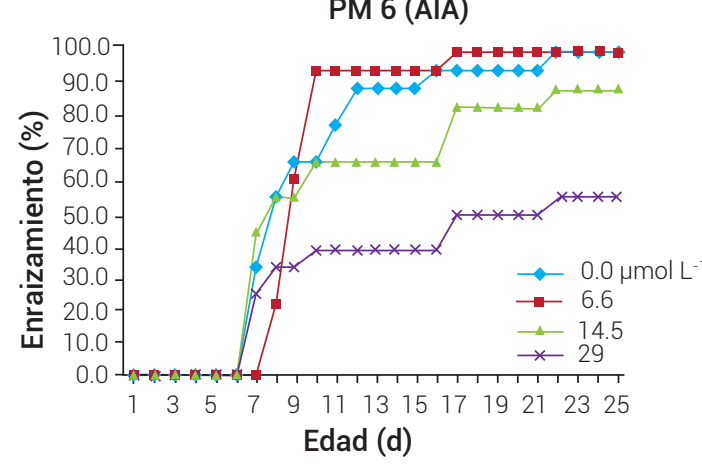

B)

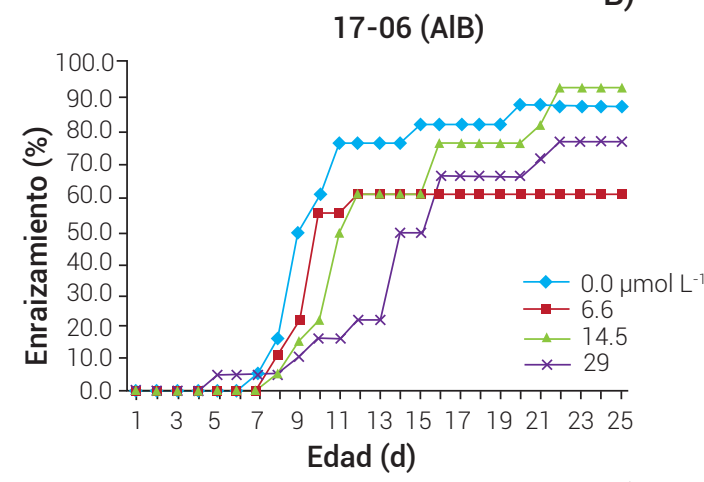

D)

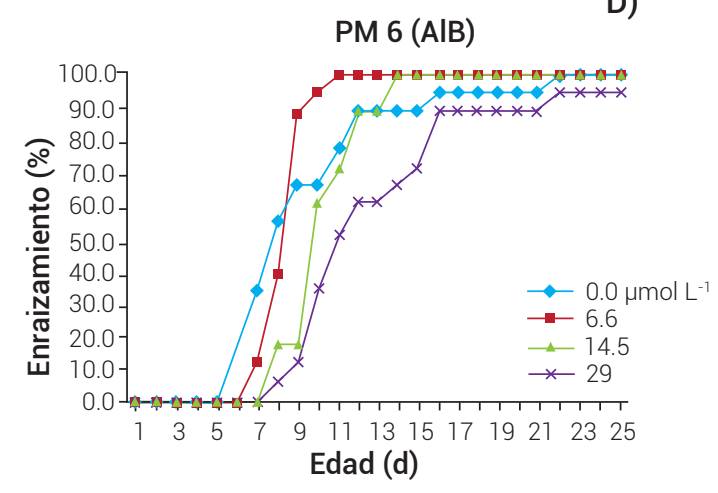

Figura 4. Efecto del tipo y concentración de auxina sobre la cinética de enraizamiento de brotes de guayabo de los genotipos 17-06 (A y B) y PM6 (C y D). 

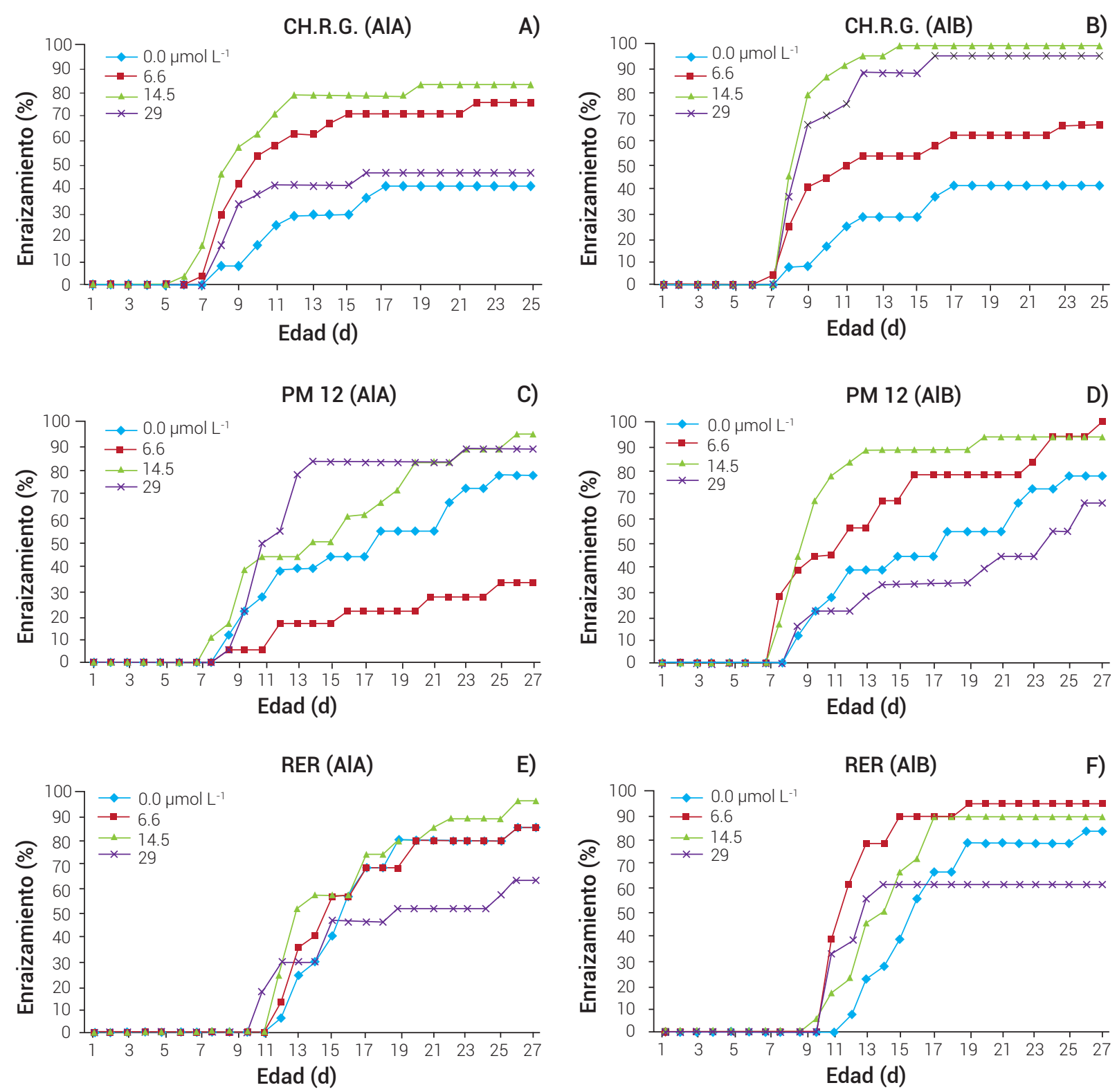

Figura 5. Efecto del tipo y concentración de auxina sobre la cinética de enraizamiento de brotes de guayabo de los genotipos CHRG (A y B), PM12 (C y D) y RER (E y F).

en donde el desarrollo de las raíces para el cv. Allahabab Safed se dio a los 17 d en una concentración de $10 \mathrm{mg} \mathrm{L}^{-1}$ $\left(49.2 \mu \mathrm{mol} \mathrm{L}^{-1}\right)$ de AlB y a los 30 d con una concentración de $25 \mathrm{mg} \mathrm{L}^{-1}\left(123 \mu \mathrm{mol} \mathrm{L}^{-1}\right)$, lo que confirma la importancia del factor genotipo.

La respuesta que tuvieron los genotipos en presencia de AIA y AIB sugiere que el tiempo de inducción de $24 \mathrm{~h}$ fue suficiente para conseguir buenos resultados, ya que el desarrollo de las raíces se vio afectado por altas concentraciones de auxinas en el medio de cultivo y porque las raíces estuvieron en contacto por tiempo prolongado, como lo reportaron Mishra et al. (2007b) con tiempos de emergencia de raíces mayores de $30 \mathrm{~d}$.

\section{CONCLUSIONES}

El uso de polivinilpirrolidona en una concentración de $500 \mathrm{mg} \mathrm{L}^{-1}$ como antioxidante limitó la pérdida de explantes de guayabo, con un valor máximo de $30 \%$ de explantes con alta fenolización para el genotipo PM6, independientemente del tipo de explante utilizado.

Los genotipos tuvieron un comportamiento diferente en las tres fases de su propagación in vitro evaluadas y su desarrollo varió con el medio de cultivo y con los reguladores 
de crecimiento utilizados. Durante la fase de multiplicación, la adición de $0.5 \mathrm{mg} \mathrm{L}^{-1}$ de bencilaminopurina generó mayores coeficientes de multiplicación para los genotipos Chapeada redonda grande, Paluma12 y Paluma 6.

La adición de precursores de citocininas favoreció el crecimiento y desarrollo de los brotes de los cinco genotipos. La adenina en una concentración de $30 \mathrm{mg} \mathrm{L}^{-1}$ promovió un mayor coeficiente de multiplicación para los genotipos PM6 y PM12, mientras que el genotipo 17-06 tuvo una mejor respuesta en $15 \mathrm{mg} \mathrm{L}^{-1}$. La adición de sulfato de adenina en una concentración de $40 \mathrm{mg} \mathrm{L}^{-1}$ aumentó los coeficientes de multiplicación de los genotipos CHRG y RER.

La inducción del enraizamiento se logró de manera eficaz con la adición de AIA y AIB durante 24 h. La cinética de enraizamiento mostró que el desarrollo de las raíces fue más rápido con $1.3 \mathrm{mg} \mathrm{L}^{-1}$ de AIB para los genotipos PM6 y RER, mientras que para el $c v .17-06$ la respuesta fue más rápida con $2.5 \mathrm{mg} \mathrm{L}^{-1}$ de AIA, y para los genotipos CHRG y PM12 la concentración de $3 \mathrm{mg} \mathrm{L}^{-1}$ de AIB indujo mayor respuesta.

Los resultados obtenidos resaltan la importancia de generar un protocolo de micropropagación para cada genotipo sobresaliente.

\section{BIBLIOGRAFÍA}

Abdelwahd R., N. Hakam, M. Labhilili and S. Udupa (2008) Use of an adsorbent and antioxidants to reduce the effects of leached phenolics in in vitro plantlet regeneration of faba bean. African Journal of Biotechnology 7:997-1002

Ali N., R. M. S. Mulwa, M. A. Norton and R. M. Skirvin (2003) Micropropagation of guava (Psidium guajava L.). Journal of Horticultural Science \& Biotechnology 78:739-741.

Bantawa P., O. S. Roy, P. Ghosh and T. K. Mondal (2009) Effect of bavistin and adenine sulphate on in vitro shoot multiplication of Picrorhiza scrophulariiflora Pennell.: an endangered medicinal plant of Indo China Himalayan regions. Plant Tissue Culture \& Biotechnology 19:237-245

Biswas B. K., N. Joshee, A. Yadav and A. K. Yadav (2007) Development and application of biotechnology in guava: a nutraceutical fruit. Acta Horticulturae 744:267-276.

Carvalho P. G. B., C. M. M. Machado, C. L. Moretti and M. E. N. Fonseca (2006) Hortaliças como alimentos funcionais. Horticultura Brasileira 24:397-404.

Chandra R., A. Bajpai, S. Gupta and R.K. Tiwari (2004) Embryogenesis and plant regeneration from mesocarp of Psidium guajava L. (guava). Indian Journal Biotechnology 3:246-248.

Chorilli M., G. R. Leonardi y H. R. N. Salgado (2007) Radicais livres e antioxidantes: conceitos fundamentais para aplicação em formulações farmacêuticas e cosméticas. Revista Brasileira de Farmácia 88:113-118.

Concepción 0., L. Nápoles, A. T. Pérez, M. Hernández, N. Peralta y R. Trujillo (2005) Efecto de tres antioxidantes en el cultivo in vitro de ápices de guayaba (Psidium guajava L.). Relación entre el origen del explante y el contenido de compuestos fenólicos. Cultivos Tropicales 26:33-39.
Couprie I., N. Ollat, J. P. Tandonnet, C. Poizat and J. P. Doazan (2000) In vitro rhizogenesis aptitudes of the petiole of different grapevine genotypes-comparison with hardwood cuttings. Acta Horticulturae 528:415-424.

Gaspar TH., C. Kevers, O. Faivre-Rampant, M. Crèvecoeur, CL. Pennel, H. Greppin and J. Domes (2003) Changing concepts in plant hormone action. In vitro Cellular Developmental Biology-Plant 39:85-106

Giri C. C., B. Shyamkumar and C. Anjaneyulu (2004) Progress in tissue culture, genetic transformation and applications of biotechnology to trees: an overview. Trees 18:115-135.

Krishna H. and S. K. Singh (2007) Biotechnological advances in mango (Mangifera indica L.) and their future implication in crop improvement-a review. Biotechnology Advances 25:223-243.

Malik S. K., R. Chaundhury and R. K. Kalia (2005) Rapid in vitro multiplication and conservation of Garcinia indica: a tropical medicinal tree species. Scientia Horticulturae 106:539-553

Mishra M., R. Chandra, R. Pati and A. Bajpai (2007a) Micropropagation of guava (Psidium guajava L.). Acta Horticulturae 735:155-158.

Mishra D. S., J. P. Tiwari and L. Shant (2007b) In vitro cloning of guava (Psidium guajava L.) cv. Pant Prabhat. Acta Horticuturae 735:127132

Murashige, T. and F. Skoog (1962) A revised medium for rapid growth and bioassays with tobacco tissue cultures. Physiologia Plantarum 15:473-497.

Nandagopal S. and B. D. R. Kumari (2006) Adenine sulphate induced high frequency shoot organogenesis in callus and in vitro flowering of Cichorium intybus L. CV. Focus - a potent medicinal plant. Acta Agriculturae Slovenica 87:415-425

Ocampo F. y V. M. Núñez (2007) Propagación in vitro de Psidium guajaba mediante organogénesis directa a partir de segmentos nodales. Revista Corpoica-Ciencia y Tecnología Agropecuaria 8:22-27.

Padilla R. J., G. E. González, V. F. Esquivel, S. E. Mercado, D. S. Hernández y P. N. Mayer (2002) Caracterización de germoplasma sobresaliente de guayabo de la región Calvillo-Cañones, México. Revista Fitotecnia Mexicana 25:393-399.

Papadatou P., C. A. Pontikis, E. Ephtimiadou and M. Lydaki (1990) Rapid multiplication of guava seedlings by in vitro shoot tip culture. Scientia Horticulturae 45:99-103

Pati P. K., S. P. Rath, M. Sharma, A. Sood and P. S. Ahuja (2006) In vitro propagation of rose-a review. Biotechnology Advances 24:94-114.

Perales A.L., H. E. Silos, L.L. Lorenzo, C.S. Perales y S.B. Flores (2016) Propagación in vitro de guayaba (Psidium guajava L.) a partir de segmentos nodales. Revista Mexicana de Ciencias Agrícolas 7:375-386.

Pérez A. T., L. Nápoles, 0. Concepción y R. Trujillo (2002) Multiplicación in vitro de brotes de guayaba (Psidium guajava L.) var. Enana Roja Cubana EEA 18-40 obtenidos a partir de semillas. Cultivos Tropicales 23:57-61.

Rai M. K., N. Akhtar and V. S. Jaiswal (2007) Somatic embryogenesis and plant regeneration in Psidium guajava L. CV. Banarasi local. Scientia Horticulturae 113:129-133.

Rai M. K., P. Asthana, V. S. Jaiswal and U. Jaiswal (2010) Biotechnological advances in guava (Psidium guajava L.): recent developments and prospects for further research. Trees 24:1-12.

SAGARPA. Secretaría de Agricultura, Ganadería, Desarrollo Rural, Pesca y Alimentación (2014) http://www.siap.gob.mx/.

Singh S. K., P. R. Meghwal, H. C. Sharma and S. P. Singh (2002) Direct shoot organogenesis on hypocotyl explants from in vitro germinated seedlings of Psidium guajava L. CV. Allahabad Safeda. Scientia Horticulturae 95:213-221

Thomas P. and J. W. Schiefelbein (2001) Combined in vitro and in vivo propagation for rapid multiplication of grapevine cV. Arka Neelamani HortScience 36:1107-1110.

Van Staden J., C. W. Fennell and N. J. Taylor (2006) Plant stress in vitro: the role of phytohormones. Acta Horticulturae 725:55-62.

Wróblewska K. (2012) The influence of adenine and benzyladenine on rooting and development of Fuchsia hybrida cuttings. Acta Agrobotanica 65:101-108. 